\title{
Reproduction Numbers and Thresholds in Stochastic Epidemic Models I. Homogeneous Populations
}

\author{
JOHN A. JACQUEZ \\ Departments of Physiology and Biostatistics, The University of Michigan, \\ Ann Arbor, Michigan \\ AND \\ PHILIP O'NEILL \\ Department of Mathematics, University of Nottingham, Nottingham NG7 2RD, England
}

Received 20 Novemher 1990; renised 3 May 1991

\begin{abstract}
We compare threshold results for the deterministic and stochastic versions of the homogeneous SI model with recruitment, death due to the disease, a background death rate, and transmission rate $\beta c X Y / N$. If an infective is introduced into a population of susceptibles, the basic reproduction number, $R_{0}$, plays a fundamental role for both, though the threshold results differ somewhat. For the deterministic model, no epidemic can occur if $R_{0} \leqslant 1$ and an epidemic occurs if $R_{0}>1$. For the stochastic model we find that on average, no epidemic will occur if $R_{0} \leqslant 1$. If $R_{0}>1$, there is a finite probability, but less than 1 , that an epidemic will develop and eventuate in an endemic quasi-equilibrium. However, there is also a finite probability of extinction of the infection, and the probability of extinction decreases as $R_{0}$ increases above 1 .
\end{abstract}

\section{INTRODUCTION}

A fundamental concept that has come out of the deterministic mathematical theory of epidemics is that of the basic reproduction number [2-4, $18,19,24,25,35]$. The basic reproduction number is the number of cases generated by one infective over the period of infectivity when that infective is introduced into a large population of susceptibles.

More precisely, the basic reproduction number is defined as follows. Let $c$ be the average number of persons contacted per person per unit timc, and let $\beta$ be the probability of transmission per contact between a susceptible and an infected. The combined parameter $\lambda=c \beta$ has units time ${ }^{-1}$ and is called the number of cffective contacts per person per unit time. Let $D$ be the mean duration of the infectious period. Then the number of contacts 
effective in transmission per infective if all contacts are with susceptibles is $R_{0}$, the basic or initial reproduction number, where

$$
R_{0}=c \beta D
$$

Note that $R_{0}$ is a dimensionless number.

If the population is a large population of susceptibles and we introduce one infective who is just beginning the infectious period, $R_{0}$ must be greater than 1 for an epidemic to take off.

$$
c \beta D-1>0
$$

Thus we have the concept of a threshold for epidemic takeoff directly related to the basic reproduction number. If $R_{0}>1$, an epidemic starts; then as the fraction of susceptibles decreases the epidemic slows, more so if those who recover are immune to the disease. If new susceptibles are introduced at a constant rate, an endemic steady state can occur when

$$
R=R_{0} S=1
$$

Here $S$ is the fraction of susceptibles in the population. $R$ has also been called the reproductive number or replacement number [24, 25]. Note that $R$ changes as the fraction of susceptibles changes.

Macdonald [35] first introduced the term reproduction rate for this quantity, although the existence of thresholds that are directly related to the reproduction rate had been demonstrated earlier by Kermack and McKendrich [31] and by Ross [44]. Dietz [18, 19] used the term reproduction rate for $R$. Anderson and May used intrinsic reproductive rate for $R_{0}$ [3] and also basic reproductive rate for $R_{0}$ and effective reproductive rate for $R$ [4]. Hethcote [24, 25] used the term replacement number for $R$ and contact number for $R_{0}$.

This simple idea has had a profound effect on epidemic theory. It appears to be a global insight that cuts through the details of the transmission process. However, it originated from consideration of deterministic models of homogeneous populations with random mixing. In a previous paper, Jacquez et al. [29] summarized the work on homogeneous populations, and Simon and Jacquez [47] examined the extensions of this idea to deterministic models of heterogeneous populations with random and nonrandom mixing. In this paper we examine its extension to the stochastic versions of the deterministic models for sexually transmitted diseases in homogeneous populations that are summarized in [29].

A considerable literature has accumulated on stochastic epidemic theory. The definitive review covering work published up to about 1974 is Bailey's book [6]. The review by Lefèvre [33] introducing the proceedings of 
the Luminy conference [20], and the other papers in that volume give a good picture of the present status of the field.

Comparing corresponding deterministic and stochastic models, one can say in general that if the model is linear, the deterministic equations are the same as the equations for the means of the stochastic model, so the two have the same solutions [6, 36, 38]. The stochastic model is then a linear birth-and-death model. That is not true for nonlinear models; then the stochastic mean number of infecteds usually falls below the number of infecteds for the deterministic model [6]. However, for many nonlinear models, for any finite interval $[0, t]$, the equations converge on those of a birth-and-death process as the initial size of the population, $N$, increases $[7,12]$. The simulations on our model show that property very nicely.

A major part of the work on stochastic epidemic models has been on the general stochastic epidemic, a name given by Bailey [5] to the stochastic version of Kermack and McKendrick's deterministic model [31]. The Kermack-McKendrick model is an SI model with removals from the infected class but no competing causes of death and no recruitment into the population, so the population is bounded. More important, the rate at which susceptibles are converted into infectives is given by a quadratic expression, $\lambda X Y$, in which $X$ and $Y$ are the numbers of susceptibles and infecteds, respectively. Commonly, $\lambda$ is treated as a constant. That differs from our models $[29,47]$ in which the rate at which susceptibles are converted into infecteds is given by a homogeneous rational expression of degree 1 in the susceptibles and the infecteds. There is now a considerable body of work on finding solutions for the probabilities, $p_{x y}(t)$, that the population has $x$ susceptibles and $y$ infectives at time $t[14,21,22,32,46$, 48], the maximum size [10,16], the final size [34], and the duration of the epidemic [11]. Metz [39] examined the model when there are several classes of infectives, and Ball [8] looked at several classes of susceptibles; also see [6] and [20].

There has also been considerable work on deriving thresholds for the general stochastic epidemic. For the linear birth-and-death process approximation, that is, $N$ large, let $Y_{0}$ be the initial number of infectives and $k$ the removal rate constant for removal of infectives. Then, if $k / \lambda<N$, the probability of extinction of the infection is $(k / \lambda N)^{Y_{0}}$; if $k / \lambda \geqslant N$, the probability of extinction is 1 . Whittle [51] and Williams [52] obtain somewhat different versions of the threshold theorem for large $N$. Ball [7] uses the convergence to a linear birth-and-death process to obtain similar results. Also see Martin-Löf [37].

Less work has been done on the general epidemic with recruitment, and that primarily in the context of modeling recurrent epidemics such as measles. Bartlett [13] compared deterministic and stochastic general epidemics with recruitment. Ridler-Rowe [43] worked with the general epi- 
demic with recruitment of susceptibles and infectives and with deaths of infectives only. Stirzaker [49] used the same model but with recruitment only into the susceptible class. Again, Bailey symmarizes work up to about 1974 [6].

The work on the general epidemic model has generated a useful body of theory and methods. However, there are a couple of problems with the basic assumptions of the model whose full import have not always been appreciated. One concerns the rate at which susceptibles are converted into infectives. That rate is $\lambda X Y$, and $\lambda$ is usually treated as a constant. We can rewrite the rate in the form $\lambda X Y=(\lambda N) X(Y / N)=(\lambda N) Y(X / N)$. Interpreting that expression, each susceptible makes $\lambda N$ contacts per unit time that transmit, the fraction $Y / N$ being with infectives, or alternatively, each infective makes $\lambda N$ transmitting contacts per unit time, but only the fraction $X / N$ are with susceptibles. Mollison [42] points out that the assumption that $\lambda$ is constant makes $R_{0}$ proportional to $N$. But it seems unreasonable to assume that the contact rate increases with $N$. It is more likely that the transmitting contact rate becomes constant for populations above some size. Anderson [1] presents data on childhood diseases that show that contact rates can be only weakly dependent on the population size, and Schenzle and Dietz. [45] conclude that contact rates are independent of population size. Hethcote and Van Ark [25] and de Palma and Lefèvre [17] discuss the implications of model formulation with $\lambda$ set constant or a function of $1 / N$.

In the general epidemic model $\lambda$ is assumed constant and the population size $N$ remains constant as the epidemic process unfolds for any particular population. Thus for any particular run, $\lambda$ and $\lambda N$ are constant. However, for populations with different $N$, one may index the populations with $N$ and have $\lambda_{N}$ change with $N$. The asymptotic results on the general epidemic model, such as those of [7] and [11], depend on making $\lambda_{N}$ proportional to $1 / N$.

Another problem is that the general epidemic model does not distinguish between the effects of removal by death and those of removal by recovery with immunity. The dynamics of the epidemic process differ markedly depending on whether or not there are deaths due to the disease and on whether or not immune individuals appear.

In this paper we examine deterministic and stochastic formulations for the SI, SIS, SIR, and SIRS models for homogeneous populations; the dynamics for the deterministic versions is summarized in Jacquez et al. [29]. These models differ from the general epidemic in that there are recruitment of susceptibles, deaths due to the disease, and competing causes of death. In addition, the assumption on contacts is that the contact rate per person, $c$, is constant. If $\beta$ is the probability of transmission per contact between an infected and a susceptible, the infection rate is then $\beta c X Y / N$, 
so that $\lambda=\beta c / N$. In relation to that, we refer the reader to an interesting paper by Gani and Purdue [23] on the general stochastic epidemic but with a generalized infection rate, $f(X, Y)$, replacing $\lambda X Y$. Gani and Purdue apply Whittle's stochastic threshold theorem to this more general form of the general stochastic epidemic. Furthermore, in our model $N$ does not remain constant as the epidemic unfolds because there are deaths due to the disease. In addition, the SIR and SIRS models clearly distinguish between the effects of death due to the disease and recovery with immunity.

The development is given in some detail for an SI model that is a simplified version of the model we have used to model the spread of HIV [28]. One of our purposes here is to provide background on the properties of the stochastic model in homogeneous populations before going on to heterogeneous populations. Stochastic modeling of the spread of HIV has really just begun. See Isham [26] for a review of AIDS modeling up to 1988. Tan and Hsu [50] used a modification of the general stochastic epidemic model for a homogeneous population to model spread in a homosexual population. They use the assumption that the number of susceptibles is very large and can be treated as a deterministic rather than a stochastic quantity. Mode et al. [40, 41] use Monte Carlo simulation to show that for a nonlincar stochastic AIDS model the cxpected number of infectives is not as large as is given by the deterministic model. Blanchard et al. [15] introduced modeling of an AIDS epidemic on a random graph.

\section{THE HOMOGENEOUS SI MODEL}

\section{MODEL}

The basic SI model with recruitment into the susceptible class, competing deaths, and deaths due to the disease is diagrammed in Figure 1. This state-transition or compartmental diagram describes the deterministic and stochastic models.

\section{THE DETERMINISTIC MODEL}

\section{Notation}

$X, Y$ the number of susceptibles and infecteds, respectively. Both are continuous, nonnegative variables.

$U$ a constant rate of recruitment of new susceptibles into the population.

$\mu \quad$ the rate constant for competing deaths, assumed to be the same for susceptibles and infecteds. Thus, the rate at which susceptibles die due to all causes is $\mu X$.

$k \quad$ the rate constant for deaths due to the disease. 


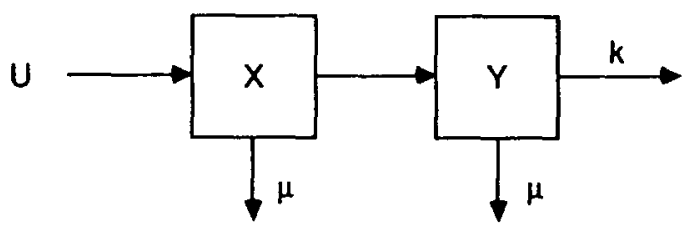

FIG. 1. State-transition diagram for the SI model with deaths due to the disease.

$c$ the mean number of persons contacted per person unit time. These contacts are, by definition, the type of contacts that can potentially transmit the disease.

$\boldsymbol{\beta}$ the probability of transmission of the disease for a contact of a susceptible with an infected.

The Deterministic Equations. The total number of persons contacted per unit time by all susceptibles is $c X$. Assuming that the contacts are randomly distributed over susceptibles and infecteds in the population, the fraction $Y /(X+Y-1)$ of these contacts is with infecteds. In the deterministic theory, it is standard to use $Y /(X+Y)$ in place of $Y /(X+Y-1)$ because $X$ and $Y$ are continuous variables. It makes little difference for $X+Y$ large, and the use of $Y /(X+Y-1)$ leads to difficulties for $X+Y \leqslant$ 1. For comparison with the stochastic model in which $X$ and $Y$ are counted in integral units, we use $Y /(X+Y-1)$ in the deterministic model and avoid the region $X+Y \leqslant 1$. Then, since $\beta$ is the fraction of contacts between susceptibles and infecteds in which there is transmission, the rate at which susceptibles are infected must be

$$
c \beta \frac{X Y}{X+Y-1} .
$$

Thus the differential equations for $X$ and $Y$ are

$$
\frac{d X}{d t}=-c \beta \frac{X Y}{X+Y-1}-\mu X+U
$$

and

$$
\frac{d Y}{d t}-c \beta \frac{X Y}{X+Y-1}-(k+\mu) Y
$$

Global Stability and the Basic Reproduction Number. If we use $X+Y$ instead of $X+Y-1$ in the denominators of the first terms in (1) and (2), Equation (2) becomes

$$
\frac{d Y}{d t}=c \beta \frac{X Y}{X+Y}-(k+\mu) Y .
$$


Then if

$$
R_{0}=\frac{c \beta}{k+\mu}<1
$$

the disease-free equilibrium is globally stable [44]. That result is obtained directly by factoring ( $2 a$ ) as in

$$
\frac{d Y}{d t}=\left[c \beta \frac{X}{X+Y}-(k+\mu)\right] Y
$$

and noting that since $X /(X+Y)<1$ for $Y>0$, if $c \beta-(k+\mu) \leqslant 0$, the derivative of $Y$ is always negative except at $Y=0$.

The result for Equation (2) is slightly different. Factoring (2),

$$
\frac{d Y}{d t}=\left[c \beta \frac{X}{X+Y-1}-(k+\mu)\right] Y
$$

At the disease-free equilibrium, $X=U / \mu, Y=0$, and

$$
\frac{X}{X+Y-1}=\frac{u}{U-\mu}
$$

Now, one obtains

$$
R_{0}=\left(\frac{c \beta}{k+\mu}\right)\left(\frac{U}{U-\mu}\right)<1
$$

as the condition for global stability of the disease-free equilibrium. Usually $U \gg \mu$, so there is little difference between the $R_{0}$ 's obtained from (3) and from (6). We continue to use the notation $R_{0}=c \beta /(k+\mu)$ and point out that the first factor in (5) gives $R_{0}$ when $Y=1$.

\section{THE STOCHASTIC MODEL}

Notation and Transitions. Now $x$ and $y$ are the numbers of susceptibles and infecteds, respectively, and are restricted in values to zero and the positive integers. The following list defines the probabilities for the various transitions; however, the terms of order $o(\Delta t)$ have been dropped because they disappear in the subsequent limit process.

$[c \beta x y /(x+y-1)] \Delta t$ is the probability that a susceptible is converted to an infected in $\Delta t$. In that transition $x$ decreases by 1 and $y$ increases by 1. This transition probability must be zero whenever $y=0$ and whenever $x=0$. In particular, whenever $x+y \leqslant 1$, there can be no transmission, so this expression has to be assigned the value zero when $x+y \leqslant 1$. 
$U \Delta t$ is the probability that $x$ increases by 1 in $\Delta t$, by recruitment.

$\mu x \Delta t$ is the probability of losing one susceptible to a competing cause of death in $\Delta t ; x$ decreases by 1 .

$\mu y \Delta t$ is the probability of losing one infective to a competing cause of death in $\Delta t ; y$ decreases by 1 .

$k y \Delta t$ is the probability of losing an infective due to the disease, in $\Delta t ; y$ decreases by 1 .

The Stochastic Equations. Define $p_{x y}(t)$ as the probability that the population has $x$ susceptibles and $y$ infectives at time $t$. We write out in full the expression for $p_{x y}(t+\Delta t)$, following the approach of Bailey [6], to show the derivation of the differential equation for $p_{x y}(t)$.

$$
\begin{aligned}
p_{x y}(t+\Delta t)= & +p_{x+1, y-1} \frac{c \beta(x+1)(y-1)}{x+y-1} \Delta t+p_{x, y+1}(k+\mu)(y+1) \Delta t \\
& +p_{x-1, y} U \Delta t+p_{x+1, y} \mu(x+1) \Delta t \\
& +p_{x y}\left[1-\frac{c \beta x y}{x+y-1} \Delta t-(k+\mu) y \Delta t-U \Delta t-\mu x \Delta t\right] . \quad(7)
\end{aligned}
$$

Rearranging and taking limits gives the differential equation for $p_{x y}$,

$$
\begin{aligned}
\frac{d p_{x y}}{d t}= & +c \beta\left[\frac{(x+1)(y-1)}{x+y-1} p_{x+1, y-1}-\frac{x y}{x+y-1} p_{x y}\right]+U\left[p_{x-1, y}-p_{x y}\right] \\
& +(k+\mu)\left[(y+1) p_{x, y+1}-y p_{x y}\right]+\mu\left[(x+1) p_{x+1, y}-x p_{x y}\right] .
\end{aligned}
$$

Note that there cannot be a negative number of susceptibles or infectives, so $p_{i j}=0$ if $i<0$ or $j<0$.

Initial Conditions. If no infectives are present, the number of susceptibles is given by a linear death process with immigration. We assume that that process is at equilibrium when the infectives are introduced. At that point, $E[x]=m_{x}=U / \mu$, so we choose for initial condition $n$ susceptibles, where $n$ is the integer closest to $U / \mu$. To such a population, we introduce $m$ infecteds. That gives for initial conditions,

$$
\begin{gathered}
p_{n m}(0)=1, \\
p_{x y}(0)=0, \quad x \neq n \text { or } y \neq m .
\end{gathered}
$$

We will be most interested in the case $m=1$.

Ranges of $x$ and $y$. Because of the recruitment, $x$ and $y$ have ranges $(0, \infty)$, but the probabilities $p_{x y}$ are infinitesimal for $x$ and $y$ much larger 
than $n$. This means that in numerical work, solving the system of ODEs (8), only a finite subset of the $p_{x y}$ are nonnegligible.

Remarks. It is worth emphasizing that Equation (8) differs considerably from the corresponding equation for the general stochastic epidemic, with or without recruitment [6]. The appearance of rational expressions in $x$ and $y$ in the first term in (8) makes it extremely difficult to obtain probabilitygenerating functions from Equation (8).

It is also worth noting that the deterministic model consists of two simultaneous nonlinear ODEs whereas the equations for the state probabilities in the stochastic version consist of an infinite set of linear ODEs.

\section{THE MEAN NUMBER OF INFECTEDS}

We will want to compare the time courses of the mean number of infecteds from the stochastic model with the time courses of the number of infecteds from the deterministic model. By definition, the expected values are given by

$$
m_{y}(t)=E[y]=\sum_{x=0}^{\infty} \sum_{y=0}^{\infty} y p_{x y}(t)
$$

and

$$
m_{x}(t)=E[x]=\sum_{x=0}^{\infty} \sum_{y=0}^{\infty} x p_{x y}(t)
$$

We have tried the generating function approach to find equations for the mean values but have failed with it. However, one can generate the differential equations for the mean values,

$$
\frac{d m_{x}}{d t}=\sum_{x=0}^{\infty} \sum_{y=0}^{\infty} x \frac{d p_{x y}}{d t}
$$

and

$$
\frac{d m_{y}}{d l}=\sum_{x=0}^{\infty} \sum_{y=0}^{\infty} y \frac{d p_{x y}}{d l}
$$

directly from the definitions of the transition probabilities.

Starting with the system in state $(x, y)$, we calculate the expected value of $y(t+\Delta t)-y(t)$,

$$
E[y(t+\Delta t)-y(t) \mid x, y]=c \beta \frac{x y}{x+y-1} \Delta t-(k+\mu) y \Delta t+o(\Delta t) .
$$


Taking expected values and using the usual limit process as $\Delta t \rightarrow 0$ gives

$$
\frac{d E[y]}{d t}=E\left[\frac{c \beta x y}{x+y-1}\right]-E[(k+\mu) y]
$$

Hence,

$$
\frac{d m_{y}}{d t}=c \beta \sum_{x=0}^{\infty} \sum_{y=0}^{\infty} \frac{x y}{x+y-1} p_{x y}-(k+\mu) \sum_{x=0}^{\infty} \sum_{y=0}^{\infty} y p_{x y} .
$$

In similar fashion one obtains

$$
\frac{d m_{x}}{d t}=-c \beta \sum_{x=0}^{\infty} \sum_{y=0}^{\infty} \frac{x y}{x+y-1} p_{x y}-\mu \sum_{x=0}^{\infty} \sum_{y=0}^{\infty} x p_{x y}+U
$$

Equations (15) and (16) can also be obtained directly by substituting (8) into (11) and (12), though with somewhat more effort.

\section{RELATIONS BETWEEN STOCHASTIC MEANS} AND DETERMINISTIC VARIABLES

1. REPRODUCTION NUMBER AND GLOBAL STABILITY: $R_{0}<1$

Rewrite Equation (15) in the form

$$
\frac{d m_{y}}{d t}=(k+\mu) \sum_{x=0}^{\infty} \sum_{y=0}^{\infty}\left[\frac{\beta c}{k+\mu}\left(\frac{x}{x+y-1}\right)-1\right] y p_{x y} .
$$

Assume that $R_{0}-1=[\beta c /(k+\mu)-1]<0$. We know the initial value, $m_{y}(0)$, is finite. Now we show that the derivative of $m_{y}(t)$ is negative for all $t<\infty$ and that its asymptotic steady value is zero.

The Derivative of $m_{y}$ is Always Negative. Consider Equation (17) for $t \geqslant 0$. All terms for which $y=0$ are equal to zero, so in the summations we need only consider the terms in $y$ for $y \geqslant 1$. For all $y \geqslant 1$ and all $x>0$,

$$
\frac{x}{x+y-1} \leqslant 1
$$

and the equality sign holds only when $y=1$ and $x \neq 0$. Hence, if $R_{0}-1<0$, all coefficients of $y p_{x y}$ in (17) must be negative for $y \geqslant 1$. Therefore the derivative of $m_{y}$ is always negative and $m_{y}$ must always decrease. 
The Equilibrium State Value of $m_{y}$ is Zero. Now we seek the equilibrium state solution for Equation (15), $m_{y}^{e}$, for which

$$
\frac{d m_{y}}{d t}=0
$$

when $R_{0}<1$. For the equilibrium state, Equation (17) can be written

$$
R_{0} \sum_{x=0}^{\infty} \sum_{y=0}^{\infty} \frac{x}{x+y-1} y p_{x y}=\sum_{x=0}^{\infty} \sum_{y=0}^{\infty} y p_{x y}-m_{y}^{e}
$$

The double sum on the left-hand side may be written

$$
\sum_{x=0}^{\infty} \sum_{y=1}^{\infty} \xi_{x y} y p_{x y}
$$

where

$$
0 \leqslant \xi_{x y} \leqslant 1
$$

Hence there exists $\xi^{e}, 0 \leqslant \xi^{e} \leqslant 1$, such that

$$
\sum_{x=0}^{\infty} \sum_{y=1}^{\infty} \xi_{x y} y p_{x y}=\xi^{e} \sum_{x=0}^{\infty} \sum_{y=1}^{\infty} y p_{x y} .
$$

Thus, (20) becomes

$$
\left(R_{0} \xi^{e}-1\right) m_{y}^{e}=0
$$

By hypothesis, $R_{0}<1$, so the first factor in (24) cannot be zero. Hence, $m_{y}^{e}=0$.

In summary, if $R_{0}-1<0$, the equilibrium state for Equation (15) has for solution $m_{y}^{e}=0$, and the derivative of $m_{y}$ is always negative. Since $y$ is a nonnegative variable, if its expected value goes to zero, all probabilities $p_{x y}$ for $y>0$ must go to zero, and hence all higher moments must also go to zero. Hence, the disease-free equilibrium is globally stable for the stochastic model. We obtain the same results as for the deterministic model.

What of the case $R_{0}=1$ ? For $x=0, \xi_{x y}=0$, and for $y=1, \xi_{x y}=1$; for all other $x$ and $y, 0<\xi_{x y}<1$. Hence, some of the coefficients in (17) are negative and others are zero. And, except for the unusual circumstance $p_{x y}(t)=0$ for $y \neq 1$, there exists $\xi^{c}, 0 \leqslant \xi^{c}<1$, such that (23) holds, so that the conclusion $m_{y}^{e}=0$ still holds. 
The Equilibrium State Value of $m_{x}$. From (16), at the steady state we obtain

$$
c \beta \xi^{e} m_{y}^{e}+\mu m_{x}^{e}=U
$$

For $R_{0}<1, m_{y}^{e}=0$, so

$$
m_{x}^{e}=U / \mu
$$

2. INITLAL TIME COURSE OF THE EPIDEMIC

For the early time course, as long as $y \ll x, p_{x y}$ will be negligible for large $y$. Then, for all terms in (15) for which $p_{x y}$ is significant,

$$
\frac{x}{x+y-1} \simeq 1
$$

Under that constraint, Equation (15) reduces to

$$
\frac{d m_{y}}{d t} \simeq[\beta c-(k+\mu)] \sum_{x=0}^{\infty} \sum_{y=0}^{\infty} y p_{x y}=[\beta c-(k+\mu)] m_{y}
$$

Initially, $m_{y}$ grows or decays exponentially; it grows if $R_{0}>1$, it decreases if $R_{0}<1$, and it is stationary for a time if $R_{0}=1$. How long this approximation holds depends on how long relation (27) is satisfied. Obviously, the larger the initial size of the population, the longer relation (28) will be valid.

With the approximation of (27) and (28), the epidemic becomes a birth-and-death process. For the general epidemic, the birth-and-death approximation holds until about $n^{1 / 2}$ of the susceptibles become infected [9]. We do not know if that holds for this model in which the size of the population decreases as the epidemic spreads.

3. ENDEMIC EQUILIBRIUM STATE: $R_{0}>1$

As we did in Equation (23), we define a mean value $\xi, 0<\xi(t)<1$, as in (29) and explicitly show its dependence on $t$.

$$
\xi(t)=\frac{\sum_{x=0} \sum_{y=1}[x /(x+y-1)] y p_{x y}}{\sum_{x=0} \sum_{y=1} y p_{x y}}
$$

$\xi(t)$ is a weighted average of $x /(x+y-1)$ with weights $y p_{x y}(t)$. Using 
(29), rewrite (15) and (16) as in

$$
\frac{d m_{y}}{d t}=(k+\mu)\left[R_{0} \xi(t)-1\right] m_{y}
$$

and

$$
\frac{d m_{x}}{d t}=-c \beta \xi(t) m_{y}-\mu m_{x}+U
$$

At the equilibrium state,

$$
\left(R_{0} \xi^{e}-1\right) m_{y}^{e}=0
$$

and

$$
m_{x}^{e}=\frac{U-(k+\mu) R_{0} \xi^{e} m_{y}^{e}}{\mu}
$$

With $R_{0}>1$, Equation (32) can in general have two solutions, but now $m_{y}^{e} \neq 0$. We note that Equation (30) has a solution for $d m_{y} / d t=0$ at which $d m_{x} / d t \neq 0$. That is the peak in the curve of $m_{y}$ shown in the simulations, Figures 5 and 6 . For the equilibrium, we obtain

$$
\begin{gathered}
\xi^{e}=1 / R_{0} \\
m_{x}^{e}=\frac{U-(k+\mu) m_{y}^{e}}{\mu}
\end{gathered}
$$

These can be compared with results on the deterministic system,

$$
\begin{gathered}
\frac{X^{s}}{X^{s}+Y^{s}}=\frac{1}{R_{0}}, \\
X^{s}=\frac{U-(k+\mu) Y^{s}}{\mu} .
\end{gathered}
$$

\section{SIMULATIONS}

In this work we have generated the state probabilities, the means, and the means conditioned on nonextinction by direct integration of the set of ODEs for the state probabilities. For simulations of the general stochastic epidemic and comparison of results with those of the linear stochastic approximation and a multivariate normal approximation, see the paper by Isham in this volume [27].

The state probabilities $p_{x y}$ were generated for $R_{0}=0.5,2.0,8.0$ and $n=10,30,100$, and 300 , with use of a SUN SPARC station, with double- 
precision arithmetic. To integrate Equations (8) for the $p_{x y}$, the $p_{x y}$ were set to zero for $x$ and for $y$ greater than certain maximum values. These maximum values for $x$ and $y$ were 30 for $n=10,70$ for $n=30$, and 140 for $n=100$; for $n=300$, the maximum value for $x$ was set to 400 , the maximum value for $y$ was 200 for $R_{0}=0.5$ and 2.0 and 300 for $R_{0}=8.0$. At each time point a check sum of the $p_{x y}$ was calculated to see that $\sum p_{x y}$ was less than 0.01 from 1.0. The check sum always started at 1.0, fell slightly to $0.9999-0.99999$, and then rose slowly above 1.0. The latter resulted from the accumulation of truncation errors in the calculations. The calculations were not accepted when the check sums rose above 1.01 .

Figures 2-4 compare the time courses of the fractions infected for the deterministic model and for the means of the stochastic SI models. In the figures, straight lines connect the plotted points; the curves are actually smooth. The curves for $n=10$ in Figures 3 and 4 do not go out to the full time scale because the check sums failed at the times the curves stop.

A number of points should be noted in Figures 2-4.
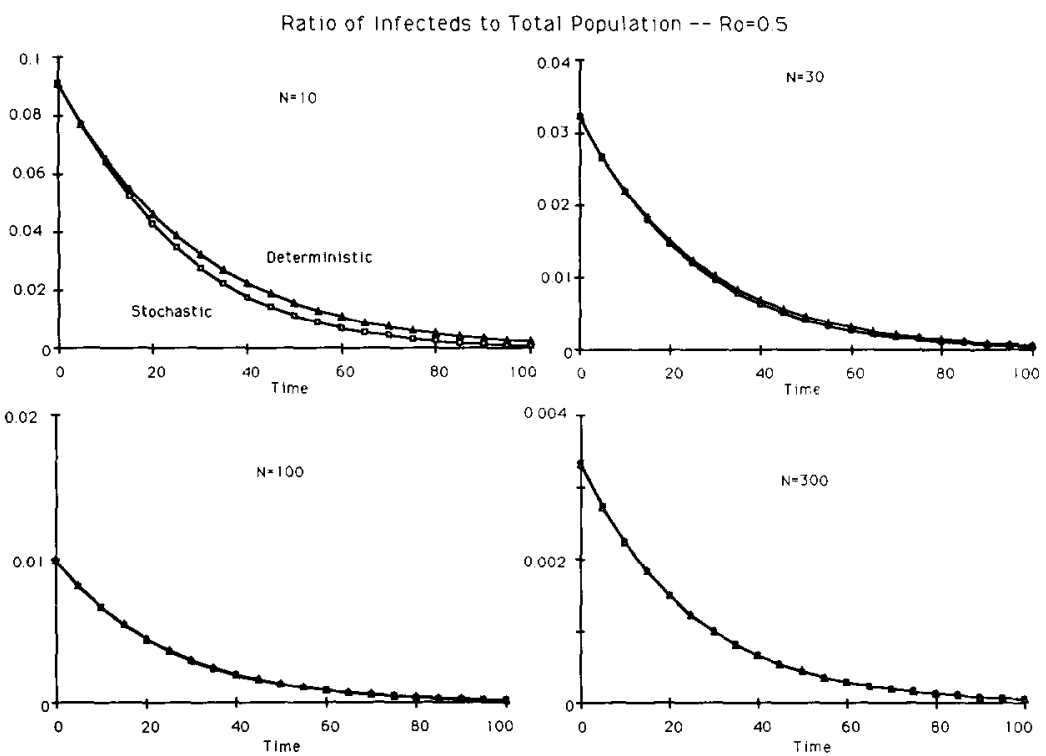

Fig. 2. Comparison of the time courses of the fraction of infecteds, $Y /(X+Y)$, for the deterministic models and $m_{y} /\left(m_{x}+m_{y}\right)$ for the stochastic models, for the SI model for $R_{0}=0.5$. The initial population sizes were $n=10,30,100,300$, respectively, for $c \beta=0.16, \mu=0.02, k=0.06$. For each case, $U=n \mu$, so $n$ is the equilibrium value for the number of susceptibles in a disease-free population. In the plotting routines, straight lines connect the plotted points. 

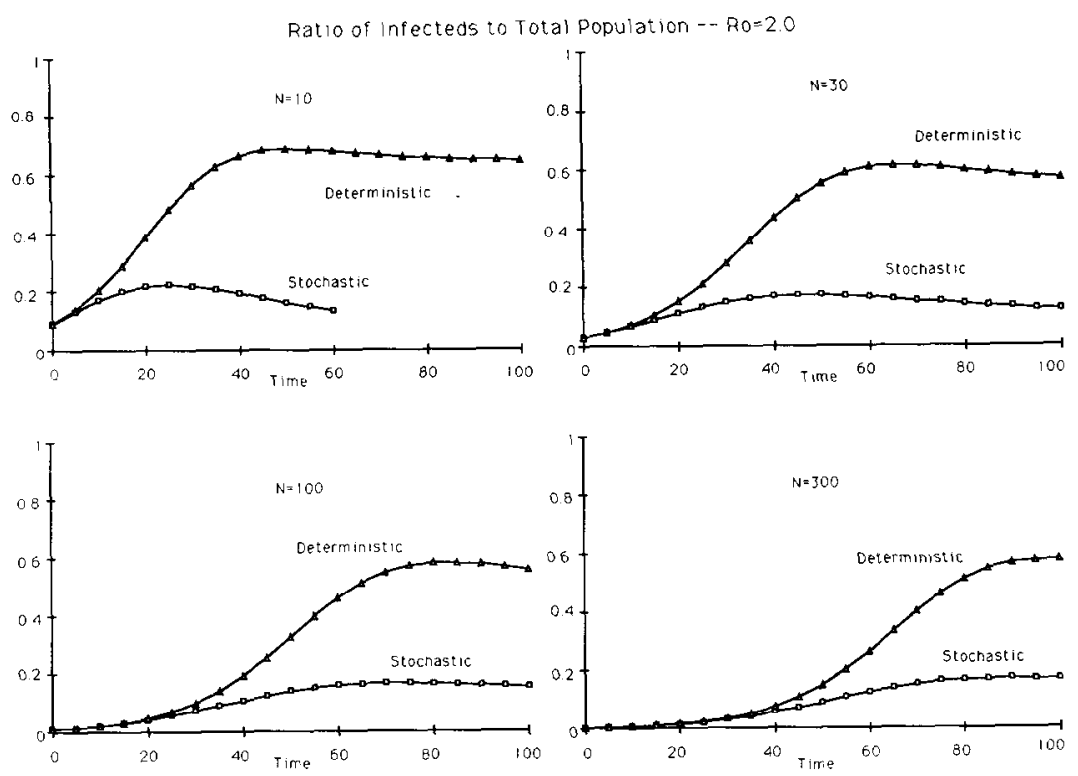

FIG. 3. Comparison of the time courses of the fraction of infecteds, $Y /(X+Y)$, for the deterministic models and $m_{y} /\left(m_{x}+m_{y}\right)$ for the stochastic models, for the SI model for $R_{0}=2.0$. See also Figure 2 .
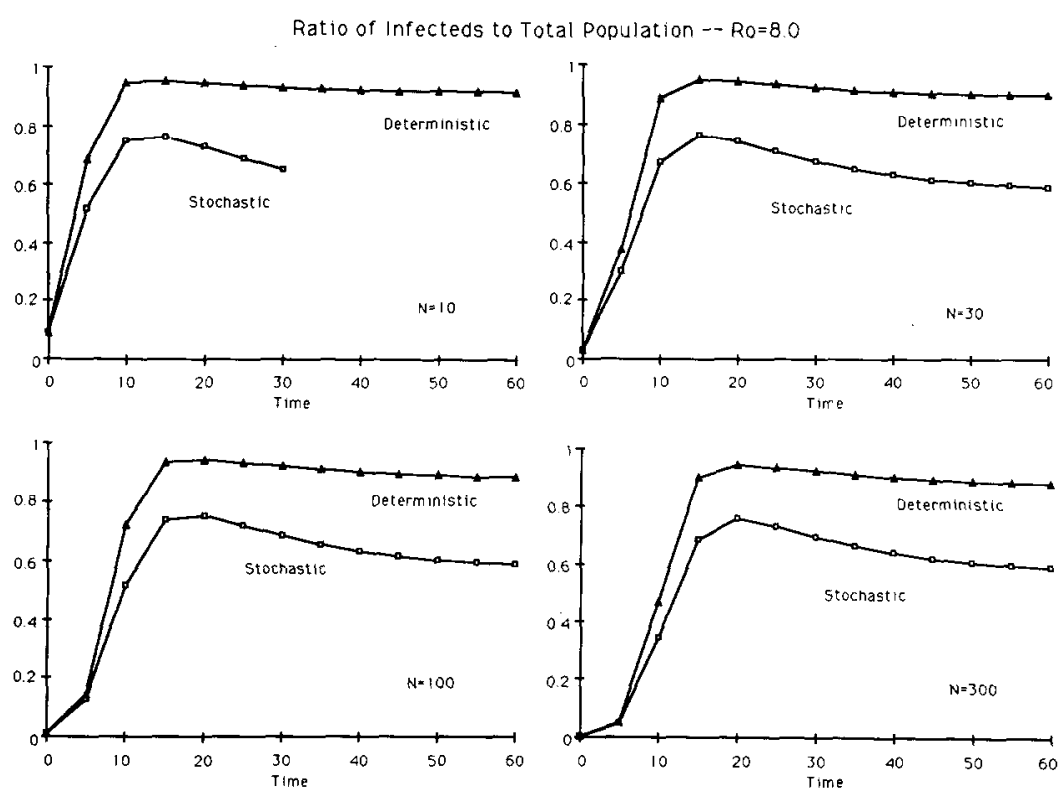

FIG. 4. Comparison of the time courses of the fraction of infecteds, $Y /(X+Y)$, for the deterministic models and $m_{y} /\left(m_{x}+m_{y}\right)$ for the stochastic models, for the SI model for $R_{0}=8.0$. See also Figure 2 . 
1. For $R_{0}=0.5$, there is a progressive fall of the deterministic ratio $Y /(X+Y)$ and of the ratio of stochastic means, $m_{y} /\left(m_{x}+m_{y}\right)$, toward zero.

2. The time courses of the corresponding deterministic and stochastic models are the same early in the process, and the length of time that holds increases with $n$. That defines the length of time the linear birth-and-death approximation holds and fits in with previous results on other stochastic models of nonlinear systems [6, 7].

3. The means for the stochastic epidemics follow time courses that are similar in shape to those of the deterministic models after the early linear phases, but the stochastic ratios fall below the deterministic ratios, in some cases well below, for example, for $R_{0}=2$. At the meeting in Skokloster someone commented that in gathering data one would perforce collect data only on realizations that gave epidemics, a point that Kendall [30] had emphasized. We note that for $R_{0}>1$ there is still a nonzero probability of extinction and that those realizations of the stochastic process in which the infection quickly died out would not be counted as epidemics. The mean $m_{y}$ is

$$
m_{y}=\frac{\sum_{x=0} \sum_{y=0} y p_{x y}}{\sum_{x=0} \sum_{y=0} p_{x y}}=\sum_{x=0} \sum_{y=0} y p_{x y} .
$$

However, only $y \geqslant 1$ contributes to the summation in the numerator. Let us define a mean for $y \geqslant 1$,

$$
m_{y}^{*}=\frac{\sum_{x=0} \sum_{y=1} y p_{x y}}{\sum_{x=0} \sum_{y=1} p_{x y}}=\frac{\sum_{x=0} \sum_{y=1} y p_{x y}}{1-\sum_{x=0} p_{x 0}} .
$$

Here, $\sum p_{x 0}(t)$ is the probability of extinction at $t$. Figures 5 and 6 compare the time courses for the deterministic number of infecteds and $m_{y}$ and $m_{y}^{*}$ for $R_{0}=2$ and $R_{0}=8$, respectively. As might be expected, $m_{y}^{*}$ is much closer to the deterministic than is $m_{y}$. However, in the early linear phase of the process where $Y$ and $m_{y}$ correspond, $m_{y}^{*}$ is higher and later crosses the deterministic time course, but by the plateau $m_{y}^{*}$ and $Y$ are very close indeed. This is more obvious for $R_{0}=2$; for $R_{0}=8$ the probability of extinction is small, so the deterministic $Y, m_{y}$, and $m_{y}^{*}$ all eventually run close together. One cannot be certain from the numerical results whether or not $Y$ and $m_{y}^{*}$ eventually become equal because of truncation errors in the calculations. The truncation errors accumulate as $t$ increases and the check sum, $\Sigma \Sigma p_{x y}$, slowly drifts above and away from 1 . Nonetheless, the results suggest the conjecture

$$
\lim _{t \rightarrow \infty}\left[m_{y}^{*}(t)-Y(t)\right]=0 .
$$



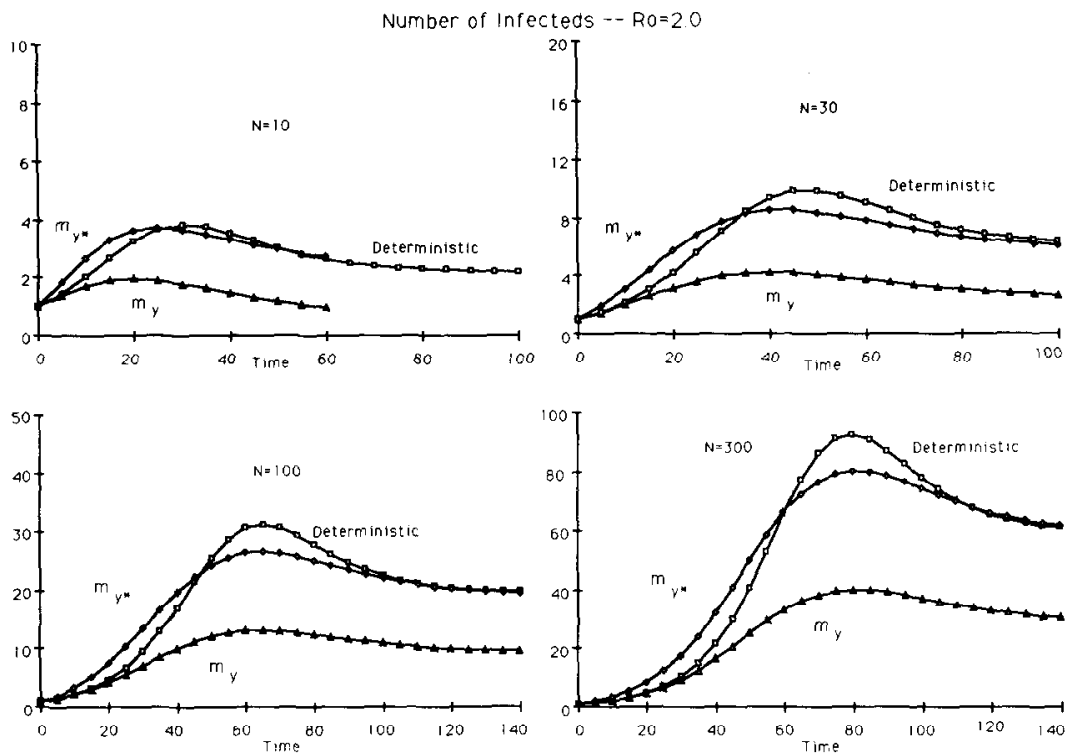

FIG. 5. Comparison of the time courses for the deterministic number of infecteds $Y$, the stochastic mean $m_{y}$, and the renormalized mean for $y \geqslant 1, m_{y}^{*}$, for $R_{0}=2$.

Number of Infecteds -- Ro $=8.0$
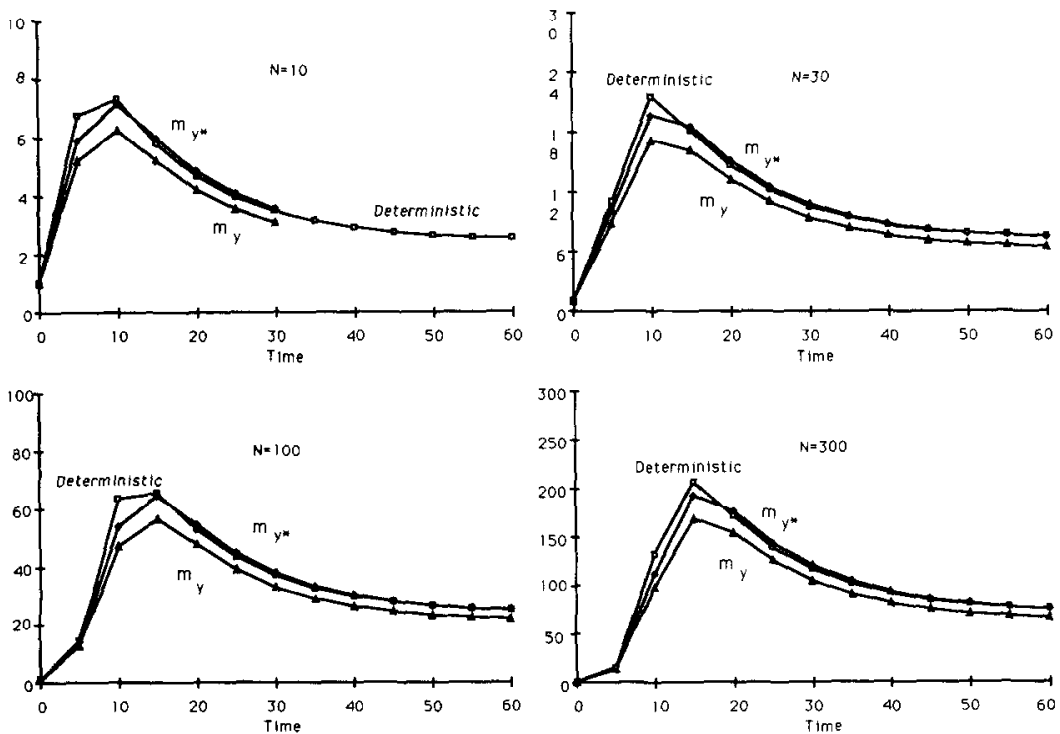

Fig. 6. Comparison of the time courses for the deterministic number of infecteds $Y$, the stochastic mean $m_{y}$, and the renormalized mean for $y, m_{y}^{*}$, for $R_{0}=8$. 


\section{SIS, SIR, AND SIRS MODELS}

The analytical results for SIS, SIR, and SIRS models are much the same as for SI models. The main results without details or derivations are given in Appendix A. However, we have not explored the SIS, SIR, and SIRS models with use of simulations.

\section{DISCUSSION}

$R_{0}$ plays much the same role in defining thresholds for epidemic takeoff in deterministic and stochastic models.

For the deterministic model, if $R_{0}<1$, the disease dies out; if $R_{0}>1$, there is an epidemic takeoff that eventually settles down to an endemic equilibrium.

The results for the stochastic models are similar but now have to be interpreted in probabilistic terms. We have shown that if $R_{0}<1$, the stochastic mean number of infecteds goes to zero. The total probability for all states can be written

$$
\sum_{x=0} p_{x 0}(t)+\sum_{x=0} \sum_{y=1} p_{x y}(t)=1
$$

The first term is the extinction probability $P_{\text {ext }}(t)$ at $t$, the second the probability of nonextinction, $P_{\text {end }}(t)$. Since $P_{\text {end }}(t) \rightarrow 0$ in $t$, for $R_{0}<1$,

$$
\lim _{t \rightarrow \infty} P_{\text {ext }}(t)=1
$$

Figure 7 shows the simulation results; $P_{\text {exi }}(t) \rightarrow 1$ and follows essentially the same time course for $n=10,30,100,300$.

For $R_{0}>1$, the stochastic mean approaches some equilibrium value.

$$
m_{y}^{e}=R_{0} \sum \sum \frac{x}{x+y-1} y p_{x y} .
$$

At the endemic equilibrium, we must have

$$
\sum_{x=0} \sum_{y=1} p_{x y} \neq 0
$$

but not 1 . There is some probability of extinction, $P_{\mathrm{ext}}^{e}<1$. Figure 7 also shows the simulation results for $P_{\text {ext }}(t)$ for $R_{0}=2$ and 8 . As $R_{0}$ increases, $P_{\text {ext }}$ decreases. For $R_{0}=2.0$, the curves for $n=100$ and 300 follow the same time course but those for $n=10$ and 30 seem to be going toward higher levels. However, the check sum hits 1.01 at $t=40$ for $n=10$ and right after $t=60$ for $n=30$, whereas the check sums for $n=100$ and 300 show a much 


$$
\text { Probability } Y=0
$$
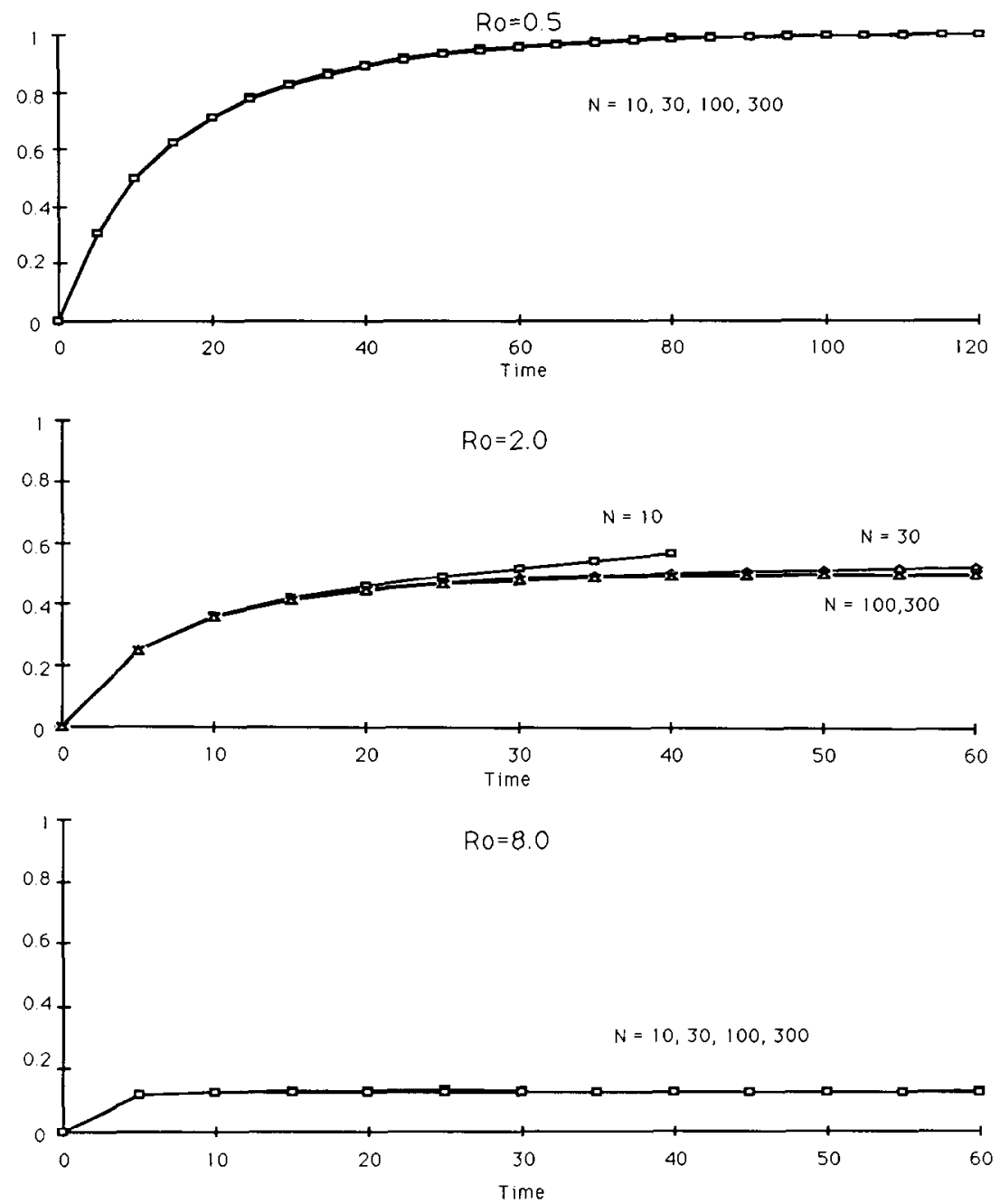

FIG. 7. Plot of the probability of extinction calculated in the simulations for $R_{0}=0.5$, $2.0,8.0$. 
lower error, so the deviations might be due to the error accumulation. The curves for $R_{0}$ also show a small deviation for $n=10$ at $t=30$ and for $n=30$ at $t=60$, again when the check sums show a $1 \%$ deviation.

The results for $R_{0}>1$ seem at first to be counterintuitive. They predict that the probability distribution for the states of the system eventually becomes stationary with a nonzero total probability, $P_{\mathrm{ext}}<1$, for the states for which $y=0$, that is, no disease present. In terms of realizations, does that mean that all realizations will die out? No, because there is always a nonzero probability associated with the states for which disease is present. For the realizations for which the disease dies out, the die-out occurs early before the epidemic has built up much. In part the counterintuitive nature comes from the difference between the distributional viewpoint and the viewpoint from realizations of the process, and the latter perhaps comes more easily to us.

The generation of a stationary distribution of state probabilities with $P_{\mathrm{end}}^{e}>0$ arises directly from the recruitment into the susceptible group. If one examines the results for the same model but without recruitment, that is, $U=0$, the results are intuitively obvious and by comparison shed light on the results for $U>0$. For $R_{0}<1$, the disease dies out in the same fashion as for $U>0$; the derivative of $m_{y}$ is always negative, so $m_{y}$ decreases monotonically to zero, from the start of the process. The results for $R_{0}>1$ are quite different. At the start, $d m_{y} / d t>0$, so the curve of $m_{y}$ increases to a peak value, but after the peak $m_{y}$ decreases progressively, and in the limit, $m_{y}=0$. In this case, the probability of extinction does eventually become 1. But note, $m_{x}$ also goes to zero; there is extinction of the population as well as the disease.

\section{APPENDIX A: RESULTS ON SIS, SIR, AND SIRS MODELS}

In this section we summarize results on SIS, SIR, and SIRS models. For the SIR and SIRS models, three subscripts are required for the probabilities, that is, $p_{x y z}$. However, the method is the same as that used with the SI model, the derivation of the differential equations differing only in details.

1. $\quad$ SIS

Figure 8 diagrams the SIS model.

Deterministic Equations

$$
\begin{gathered}
\frac{d X}{d t}=-c \beta \frac{X Y}{X+Y-1}-\mu X+\delta Y+U, \\
\frac{d Y}{d t}=c \beta \frac{X Y}{X+Y-1}-(k+\mu+\delta) Y
\end{gathered}
$$




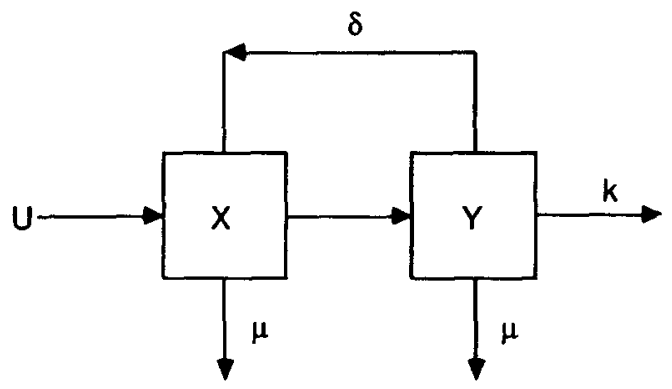

FIG. 8. State-transition diagram for the SIS model with deaths due to the disease.

Stochastic Equations. The equation for $p_{x y}$ now becomes

$$
\begin{aligned}
& \frac{d p_{x y}}{d t}=+c \beta\left[\frac{(x+1)(y-1)}{x+y-1} p_{x+1, y-1}-\frac{x y}{x+y-1} p_{x y}\right] \\
& +U\left[p_{x-1, y} p_{x y}\right]+\delta\left[(y+1) p_{x-1, y+1} \quad y p_{x y}\right] \\
& +(k+\mu)\left[(y+1) p_{x, y+1}-y p_{x y}\right]+\mu\left[(x+1) p_{x+1, y}-x p_{x y}\right] \text {. }
\end{aligned}
$$

The differential equation for $m_{y}$ becomes

$$
\frac{d m_{y}}{d t}=\sum_{x} \sum_{y}\left[\frac{\beta c x}{x+y-1}-(k+\mu+\delta)\right] y p_{x y} .
$$

As might be expected, (A4) is the same as the corresponding equation for the SI model, with the addition of the term due to the transition back to susceptibility.

The results are the same as for the SI model, but with $R_{0}=\beta c /(k+$ $\mu+\delta$ ). The disease-free equilibrium is globally stable if $R_{0}-1<0$.

2. $S I R$

Figure 9 diagrams the transitions for the SIR model.

Equations for the Deterministic Model

$$
\begin{gathered}
\frac{d X}{d t}=-c \beta \frac{X Y}{X+Y+Z-1}-\mu X+U, \\
\frac{d Y}{d t}=c \beta \frac{X Y}{X+Y+Z-1}-(k+\mu+\gamma) Y, \\
\frac{d Z}{d t}=\gamma Y-\mu Z .
\end{gathered}
$$




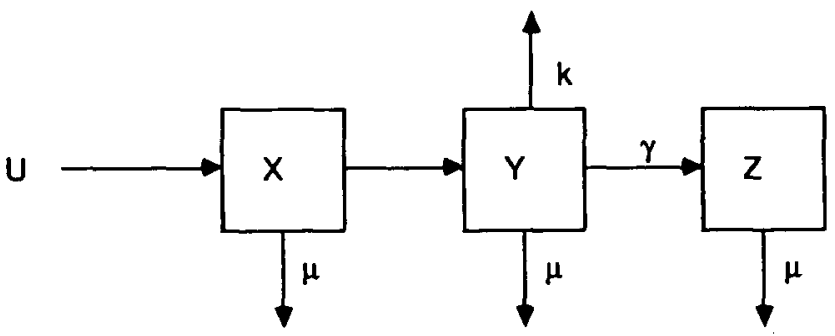

FIG. 9. State-transition diagram for the SIR model with deaths due to the disease.

Equations for the Stochastic Model

$$
\begin{aligned}
\frac{d p_{x y z}}{d t}= & +c \beta\left[\frac{(x+1)(y-1)}{x+y+z-1} p_{x+1, y-1, z}-\frac{x y}{x+y+z-1} p_{x y z}\right] \\
& +U\left[p_{x-1, y z}-p_{x y z}\right]+\mu\left[(x+1) p_{x+1, y z}-x p_{x y z}\right] \\
& +(k+\mu)\left[(y+1) p_{x, y+1, z}-y p_{x y z}\right]+\mu\left[(z+1) p_{x y, z+1}-z p_{x y z}\right] \\
& +\gamma\left[(y+1) p_{x, y+1, z+1}-y p_{x y z}\right] .
\end{aligned}
$$

Now define $m_{y}$ as

$$
m_{y}=\sum_{x} \sum_{y} \sum_{z} y p_{x y z}
$$

Its differential equation becomes

$$
\frac{d m_{y}}{d t}=\sum_{x} \sum_{y} \sum_{z}\left[\frac{\beta c x}{x+y+z-1}-(k+\mu+\gamma)\right] y p_{x y z} .
$$

Again, the results on thresholds are the same as for the SI and SIS models, but now $R_{0}=\beta c /(k+\mu+\gamma)$. Note that the presence of immunes makes $d m_{y} / d t$ more negative. 


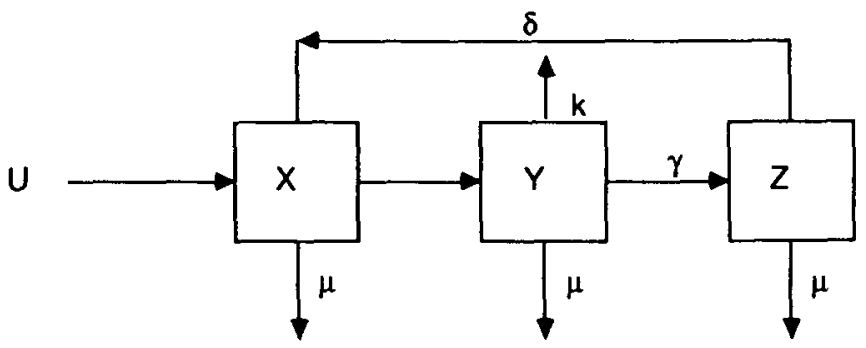

FIG. 10. State-transition diagram for the SIRS model with deaths due to the disease.

3. SIRS

Figure 10 diagrams the SIRS model.

Equations for the Deterministic Model

$$
\begin{gathered}
\frac{d X}{d t}=-c \beta \frac{X Y}{X+Y+Z-1}-\mu X+U+\delta Z \\
\frac{d Y}{d t}=c \beta \frac{X Y}{X+Y+Z-1}-(k+\mu+\gamma) Y \\
\frac{d Z}{d t}=\gamma Y-(\mu+\delta) Z
\end{gathered}
$$

Equations for the Stochastic Model

$$
\begin{aligned}
\frac{d p_{x y z}}{d t}= & +c \beta\left[\frac{(x+1)(y-1)}{x+y+z-1} p_{x+1, y-1, z}-\frac{x y}{x+y+z-1} p_{x y z}\right] \\
& +U\left[p_{x-1, y z}-p_{x y z}\right]+\mu\left[(x+1) p_{x+1, y z}-x p_{x y z}\right] \\
& +(k+\mu)\left[(y+1) p_{x, y+1, z}-y p_{x y z}\right]+\mu\left[(z+1) p_{x y, z+1}-z p_{x y z}\right] \\
& +\gamma\left[(y+1) p_{x, y+1, z+1}-y p_{x y z}\right]+\delta\left[(z+1) p_{x-1, y, z+1}-z p_{x y z}\right] .
\end{aligned}
$$

The equation for the derivative of $m_{y}$ is the same as for the SIR model, and the threshold results are the same. 
This work was supported in part by grant RR02176-01A1 from NIH-DRR, $D H E W$, by grant R01 AI29876 from NLAID, DHEW, and in part by a Presidential Initiatives Grant from the University of Michigan. The simulations were programmed and run by Timothy J. Perry. We thank Frank Ball, Andrew Barbour, Claude Lefève, Charles Mode, and Denis Mollison for their very useful critiques of prior versions of this paper.

\section{REFERENCES}

1 R. M. Anderson, Transmission dynamics and control of infectious disease agents, in Population Biology of Infectious Diseases, R. M. Anderson and R. M. May, Eds., Springer, New York, 1982, pp. 149-176.

2 R. M. Anderson and R. M. May, Population Biology of Infectious Diseases, Dahlem Workshop Report, Springer-Verlag, New York, 1982.

3 R. M. Anderson and R. M. May, Directly transmitted infectious diseases: control by vaccination, Science 215:1053-1060 (1982).

4 R. M. Anderson and R. M. May, Vaccination against rubella and measles: quantitative investigations of different policies, J. Hyg. Camb. 90:259-325 (1983).

5 N. T. J. Bailey, The Mathematical Theory of Epidemics, Charles Griffin, London, 1957.

6 N. T. J. Bailey, The Mathematical Theory of Infectious Diseases, 2nd ed., Charles Griffin, London, 1975.

7 F. Ball, The threshold behaviour of epidemic models, J. Appl. Probab. 20:227-241 (1983).

8 F. Ball, Deterministic and stochastic epidemics with several kinds of susceptibles, Adv. Appl. Probab. 17:1-22 (1985).

9 F. Ball and P. Donnelly, Branching process approximation of epidemic models, Proc. 2nd World Congress of the Bernoulli Soc., Uppsala, 1990 (to appear).

10 A. D. Barbour, $\Lambda$ note on the maximum size of a closed epidemic, J. Roy. Stat. Soc., B 37:450-460 (1975).

11 A. D. Barbour, The duration of the closed stochastic epidemic, Biometrika 62:477-482 (1975).

12 M. S. Bartlett, Stochastic Processes, Cambridge Univ. Press, 1955.

13 M. S. Bartlett, Deterministic and stochastic models for recurrent epidemics, Proc. 3rd Berkeley Symp. on Math. Stat. and Probab., J. Neyman, Ed., Vol. IV, 1956, pp. 81-109.

14 L. Billard, Factorial moments and probabilities for the general stochastic epidemic, J. Appl. Probab. 10:277-288 (1973).

15 P. Blanchard, G. F. Bolz, and T. Kruger, Modeling AIDS epidemics or any venereal disease on random graphs, Lect. Notes Biomath. 86:104-117 (1990).

16 H. E. Daniels, The maximum size of a closed epidemic, Adv. Appl. Probab. 6:606-621 (1974).

17 A. DePalma and C. Lefèvre, Population systems with (non)-extensive interaction rates, Math. Comput. Modeling 10:359-365 (1988).

18 K. Dietz, Transmission and control of arbovirus diseases, in Epidemiology, D. Ludwig and K. L. Cooke, Eds., Proceedings of a SIMS Conference on Epidemiology, SIAM, Philadelphia, 1975, pp. 104-121. 
19 K. Dietz, Mathematical models for infectious diseases; evaluation of measures for prevention and control, in Perspectives in Medical Statistics, J. F. Bithell and R. Coppi, Eds., Academic, New York, 1981, pp. 65-84.

20 J.-P. Gabriel, C. Lefevre, and P. Picard, Eds., Stochastic Processes in Epidemic Theory (Lect. Notes in Biomath. Vol. 86), Springer-Verlag, N.Y., 1990.

21 J. Gani, On a partial differential equation of epidemic theory. I, Biometrika 52:617-622 (1965).

22 J. Gani, On the general stochastic epidemic. Proceedings 5th Berkeley Symp. on Math. Stat. and Probab., Vol. IV, Univ. California Press, Berkeley, 1967, pp. 271-279.

23 J. Gani and P. Purdue, Matrix-geometric methods for the general stochastic epidemic, IMA J. Math. Appl. Med. Biol. 1:333-342 (1984).

24 H. Hethcote, Qualitative analysis of communicable disease models, Math. Biosci. 28:335-356 (1976).

25 H. W. Hethcote and J. W. Van Ark, Epidemiological models for heterogeneous populations: proportionate mixing, parameter estimation, and immunization programs, Math. Biosci. 84:85-118 (1987).

$26 \mathrm{~V}$. Isham, Mathematical modeling of the transmission dynamics of HIV infection and AIDS: a review, J. Roy. Stat. Soc. A 151:5-30 (1988).

27 V. Isham, Assessing the variability of stochastic epidemics, Math. Biosci. (this issue).

28 J. A. Jacquez, C. P. Simon, J. Koopman, L. Sattenspiel, and T. Perry, Modeling and analyzing HIV transmission: the effect of contact patterns, Math. Biosci. 92:119-199 (1988).

29 J. A. Jacquez, C. P. Simon, and J. S. Koopman, The reproduction number in deterministic models of sexually transmitted diseases, Comments Theor. Biol. 2:159-209 (1991).

30 D. G. Kendall, Deterministic and stochastic epidemics in closed populations, Third Berkeley Symp. on Math. Stat. and Prob., Vol. IV, J. Neyman, Ed., Univ. California Press, Berkeley, 1956, pp. 149-165.

31 W. O. Kermack and A. G. McKendrick, A contribution to the mathematical theory of epidemics, Proc. Roy. Soc. London A115:700-721 (1927).

32 R. J. Kryscio, The transition probabilities of the general stochastic epidemic model, J. Appl. Probab. 12:415-424 (1975).

33 C. Lefèvre, Stochastic epidemic models for SIR infectious diseases: a brief survey of the recent general theory, Lect. Notes Biomath. 86:1-12 (1990).

34 D. Ludwig, Final size distribution for epidemics, Math. Biosci. 23:33-46 (1975).

35 G. Macdonald, The analysis of equilibrium in malaria, Trop. Dis. Bull. 49:813-829 (1952).

36 B. C. McInnis, S. A. El-Asfouri, and S. A. Kapadia, On stochastic compartmental modeling. Letter to the Editor, Bull. Math. Biol. 41:611 613 (1979).

37 A. Martin-Löf, Threshold limit theorems in the theory of rumors, snowball sampling and epidemics, Lect. Notes Biomath. 86:184-188 (1990).

38 J. H. Matis, An introduction to stochastic compartmental models in pharmacokinetics, in Pharmacokinetics (NATO ASI Series, Vol. 145), A. Pecile and A. Rescigno, Eds., Plenum, New York. 1988, pp. 113-128.

39 J. A. J. Metz, The epidemic in a closed population with all susceptibles equally vulnerable; some results for large susceptible populations and small initial infections, Acta Biotheor. 27:75-123 (1978). 
40 C. J. Mode, H. E. Gollwitzer, and N. Herrmann, A methodological study of a stochastic model of an AIDS epidemic, Math. Biosci. 92:201-229 (1988).

41 C. J. Mode, H. E. Gollwitzer, M. A. Salsburg, and C. K. Sleeman, A methodological study of a nonlinear stochastic model of an AIUS epidemic with recruitment, IMA J. Math. Appl. Med. Biol. 6:179-203 (1989).

42 D. Mollison, Simplifying epidemic models, Nature 310:224-225 (1984).

43 C. J. Ridler-Rowe, On a stochastic model of an epidemic, J. Appl. Probab. 4:19-33 (1967).

44 R. Ross and H. P. Hudson, An application of the theory of probabilities to the study of a priori pathometry. Part III, Proc. Roy. Soc. London A93:225-240 (1917).

45 D. Schenzle and K. Dietz, Critical population sizes for endemic virus transmission, in Raumliche Persistenz und Diffusion von Krankheiten, W. Fricke and E. Hinz, Eds., Heidelberger Geographische Arbeiten no. 83, Heidelberg, 1986, pp. 31-42.

46 N. C. Severo, A recursion theorem on solving differential-difference equations and applications to some stochastic processes, J. Appl. Probab. 6:673-681 (1969).

47 C. P. Simon and J. A. Jacquez, Reproduction numbers and the stability of equilibria of SI models for heterogeneous populations, SLAM J. Appl. Math. (in press).

48 V. Siskind, A solution of the general stochastic epidemic, Biometrika 52:613-616 (1965).

49 D. R. Stirzaker, A perturbation method for the stochastic recurrent epidemic, $J$. Inst. Math. Appl. 15:135-160 (1975).

50 W. Y. Tan and H. Hsu, Some stochastic models of AIDS spread, Stat. Med. 8:121-136 (1989).

51 P. Whittle, The outcome of a stochastic epidemic-a note on Bailey's paper, Biometrika 42:116-122 (1955).

52 T. Williams, An algebraic proof of the threshold theorem for the general stochastic epidemic, Adv. Appl. Probab. 3:223 (1971). 